\title{
Atrioventricular Valve Abnormalities: From Molecular Mechanisms Underlying Morphogenesis to Clinical Perspective
}

\author{
Kei Inai
}

\begin{abstract}
Malformation of the atrioventricular (AV) cushion is a common congenital heart defect. Ebstein's anomaly, characterized by a heart defect related to the AV cushion, involves not only a valve defect but also a myocardial abnormality such as Uhl's anomaly. The morphogenetic features of the heart in the case of these diseases can be used as a reference for investigating valvuloseptal and myocardial formations in the human heart.

The AV endocardium transforms into the cushion mesenchyme through epithelial-mesenchymal transition (EMT). After the EMT, distal outgrowth and maturation of endocardial cushions are important morphogenetic steps for AV valvuloseptal morphogenesis. While bone morphogenetic protein (BMP)2 is known to be critical for AV EMT, little is known about the functional relationship between BMP and ECM and their roles in cushion mesenchymal cell (CMC) migration after EMT. In our previous study, we showed that BMP-2 and BMP signaling induced AV CMC migration. We have been exploring the role of BMP-2 in the regulation of valvulogenic extracellular matrix (ECM) components, periostin, versican, and hyaluronic acid (HA), and cell migration during post-EMT AV cushion expansion and maturation.

We further examined whether BMP-2-promoted cell migration is associated with expression of periostin, versican, and HA. BMP-2-promoted cell migration is significantly impaired by treatment with versican siRNA and HA oligomer. We also found that transcription of Twist and IdI, implicated in cell migration in embryogenesis and activation of the periostin promoter, was induced by BMP-2 but repressed by noggin in CMC cultures.
\end{abstract}

\footnotetext{
K. Inai ( $\square)$

Department of Pediatric Cardiology, Heart Institute, Tokyo Women's Medical University, 8-1 Kawada-cho, Shinjuku-ku, Tokyo, Japan

e-mail: pinai@hij.twmu.ac.jp

T. Nakanishi et al. (eds.), Etiology and Morphogenesis of Congenital Heart Disease, DOI 10.1007/978-4-431-54628-3_17
} 
Taken together, we provide evidence that BMP-2 induces expression and deposition of three major ECM proteins, periostin, versican, and HA, and that these ECM components contribute to BMP-2-induced CMC migration during post-EMT AV cushion expansion and maturation.

Based on these findings, we discuss the morphogenetic process of AV valve abnormalities and crosstalk between valve and cardiomyocytes morphogenesis.

\section{Keywords}

Bone morphogenetic protein (BMP) - Cardiac cushions • Periostin • Extracellular matrix protein $\bullet$ Valvulogenesis

\subsection{Introduction}

Our institute houses a huge amount of congenital heart disease specimens, of which approximately 4,000 are referred to as Prof. Ando's collections, and the late Dr. Ando, former professor at our department, wrote 30 books describing the anatomical features of the specimens.

In one of the books, I read the following text at the edge of a page: "I think there is a right ventricle (RV)-tricuspid valve (TV) dysplastic syndrome as a new clinical entity, which comprises three abnormalities, Ebstein's anomaly, Uhl's anomaly, and tricuspid absence." This note can be likened to the one written by Fermat, on the basis of which Fermat's son Clément-Samuel deduced Fermat's last theorem (Fig. 17.1).

Fig. 17.1 Tricuspid valve anomalies syndrome or RV-TV dysplastic syndrome: Ebstein's anomaly, Uhl's anomaly and tricuspid valve absence sometimes coexist in a heart

\section{Tricuspid valve anomalies syndrome}

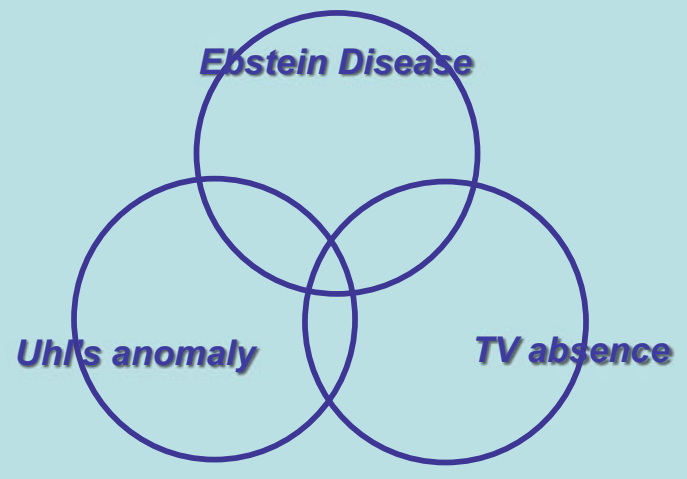




\subsection{RV-TV Dysplastic Syndrome}

\subsubsection{Anatomic Features of the Heart in Ebstein's Anomaly Patients}

Ebstein's anomaly is a rare congenital cardiac disease in which the TV leaflets are displaced to the right ventricular cavity, resulting in atrialization of the RV [1]. Marked right atrial enlargement caused by severe tricuspid insufficiency, cardiomegaly, and increased right atrial pressure; cyanosis caused by right-left shunt by way of interatrial communication, if present; and right ventricular volume overload are often observed. Figure 17.2 illustrates Ebstein's anomaly with severe $\mathrm{TV}$ insufficiency involving two-thirds of the RV.

\subsubsection{Morphogenetic Features of the Heart in Patients with Uhl's Anomaly}

In the case of patients with Ebstein's anomaly, the RV of the heart often shows partial or total loss of myocardium; in other words, the features of Ebstein's anomaly overlap those of Uhl's anomaly. Uhl's anomaly, a rare congenital heart disease characterized by the absence of apical trabeculations in the right ventricular cavity with a thin hypokinetic ventricular wall, was first described in 1952 [2]. In Fig. 17.3, almost complete absence of the right ventricular myocardium, preserved septum, and left ventricular myocardium is seen. These clinical and anatomic findings strongly indicate that $\mathrm{AV}$ valve formation and ventricular myocardium formation may play a role during cardiac morphogenesis.

Fig. 17.2 Ebstein's anomaly with severe TV plastering down into two thirds of right ventricle (RV). The cushion tissue plastered almost throughout RV with a something like plaster and it looks very smooth surface

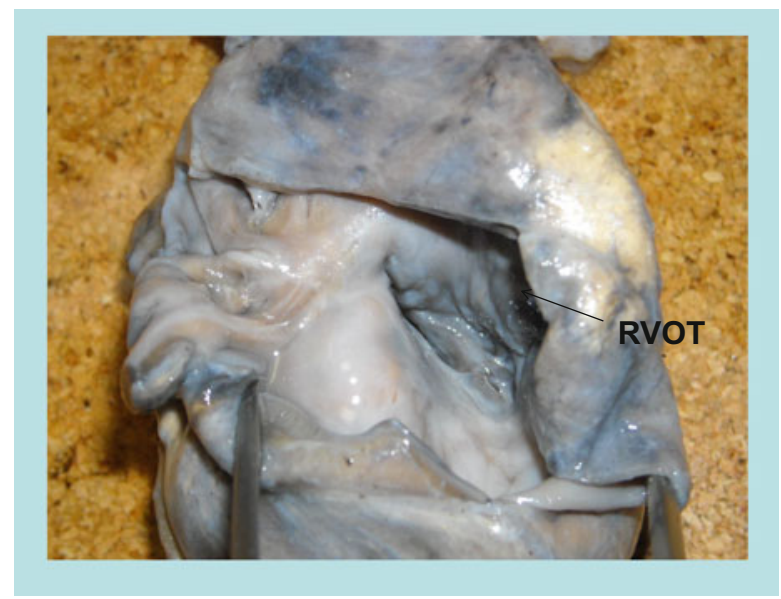


Fig. 17.3 Ebstein's anomaly with Uhl's anomaly. Note the complete absence of the right ventricular myocardium and preserved septal and left ventricular myocardium

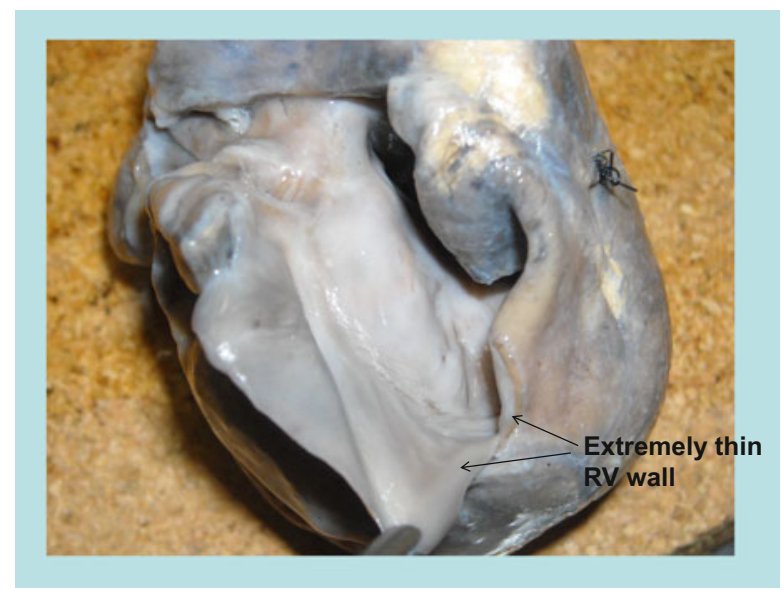

\subsubsection{Absence of the TV}

Unguarded TV orifice is an unusual cardiac malformation that involves partial or total agenesis of the TV tissue (absence of the TV). Kanjuh et al. described the anatomic features of the heart in this disease: the wall of the RV is formed by slightly trabeculated muscle and lined by thickened endocardium [3]; the free wall of the RV is only $2 \mathrm{~mm}$ thick, and the ventricular septum is intact. In Fig. 17.4, the specimen shows TV absence and partial loss of the right ventricular myocardium.

TV-RV dysplastic syndrome revealed the important link between TV morphogenesis and right ventricular myocardium formation during the biological process of cardiac morphogenesis. Therefore, it is vital to determine the biological key factors that link the myocardium and cushion tissue.

\subsection{Bone Morphogenetic Proteins (BMPs) and Their Important Role in Cushion Formation}

\subsubsection{Role of BMP2 in Cushion Mesenchymal Cell (CMC) Migration}

Anomalies related to valvuloseptal formation are some of the most common congenital heart anomalies. Two segments of the endocardium, the atrioventricular (AV) and outflow tract (OT) endocardium, transform into the cushion mesenchyme - the primordia of the valves and membranous septa - through epithelialmesenchymal transformation (EMT). Transformed endocardial cells subsequently migrate to the underlying extracellular matrix (ECM), called "cardiac jelly," and remodel the ECM into cardiac cushions [4]. Distal outgrowth and maturation of the cardiac cushion are the initial and critical morphogenetic steps in post-EMT valve formation. 


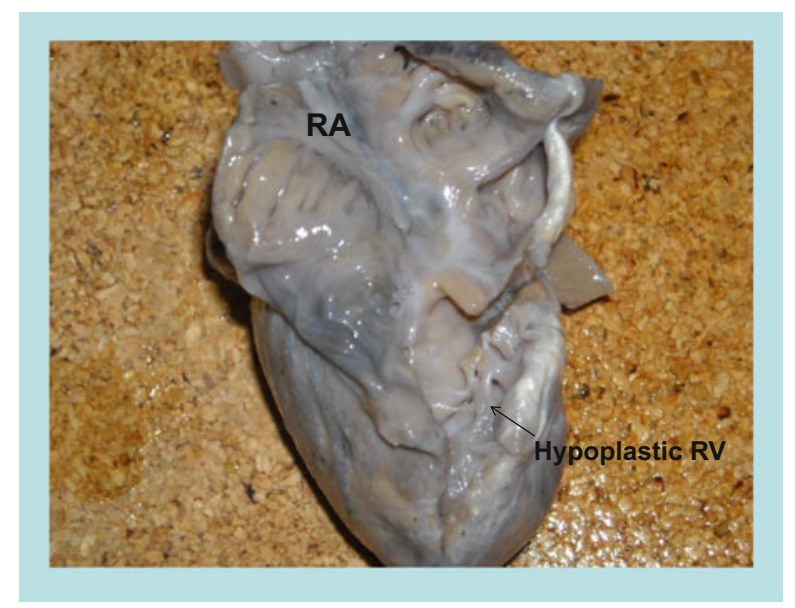

Fig. 17.4 Ebstein's anomaly with tricuspid valve absence and Uhl's anomaly. Unguarded tricuspid valve orifice and partial absence of the right ventricular myocardium concomitant with Ebstein's anomaly. Note the absence of the rudimentum of the tricuspid valve and tension apparatus

BMP is a member of the TGF- $\beta$ superfamily proteins, one of many molecules related to AV EMT. BMP signaling was found to be crucial for AV EMT in studies involving explant cultures in mice and chicks and BMP-2 conditional knockout (CKO) experiments in mice [5]. BMP-2 conditional KO at the EMT stage, however, causes subsequent lethality, thereby hindering the examination of the role of BMP-2 in post-EMT valve formation. BMPs bind to the cell surface of BMP receptors (BMPRs)-Type I and Type II receptors. Type II receptors transphosphorylate the glycine-serine-rich domain of Type I receptors and transduce intracellular signals. The Type II BMP receptor, BMPRII, is reported to be expressed ubiquitously throughout the embryonic period [6].

We previously showed the expression patterns of Type I BMPRs by localizing $B M P R-1 A(A L K 3), B M P R-1 B(A L K 6)$, and $A L K 2$ in the post-EMT AV cushion mesenchyme in a chick. These findings indicate that BMP signaling regulates the biological processes necessary for distal outgrowth and maturation of post-EMT cushion mesenchyme during early valvulogenesis [4].

\subsubsection{BMP2 Induces CMC Migration and Id and Twist Expression}

BMP-2 is localized in the AV cushion mesenchyme during post-EMT valve formation, suggesting autocrine signaling by BMP-2 in CMCs during post-EMT valve formation. Therefore, to determine the roles of BMP-2 and BMP signaling in post-EMT AV CMCs, we established a hanging-drop culture system and spatiotemporal viral gene-transfer technique using chick AV CMC cultures both in vitro and 
whole embryo cultures in vivo (ovo) [5]. Although most BMP receptors have ligandbinding affinity with other TGF- $\beta$ superfamily proteins, BMPR-1A and BMPR-1B bind specifically with BMP-2 and BMP-4 (bind with BMP-7 at low affinity) but do not bind with other TGF- $\beta$ superfamily proteins. We showed that $d n B M P R-1 B$ infection as well as treatment with a BMP antagonist, noggin, significantly inhibited endogenous phospho-Smad 1/5/8 expression in AV CMCs, indicating that intracellular BMP signaling in the CMCs is effectively inhibited by treatment with dnBMPR$1 \mathrm{~B}$ or noggin. On the other hand, BMP-2 or caBMPR-1B-virus treatment induced expression of phospho-Smad 1/5/8, indicating that intracellular BMP signaling was

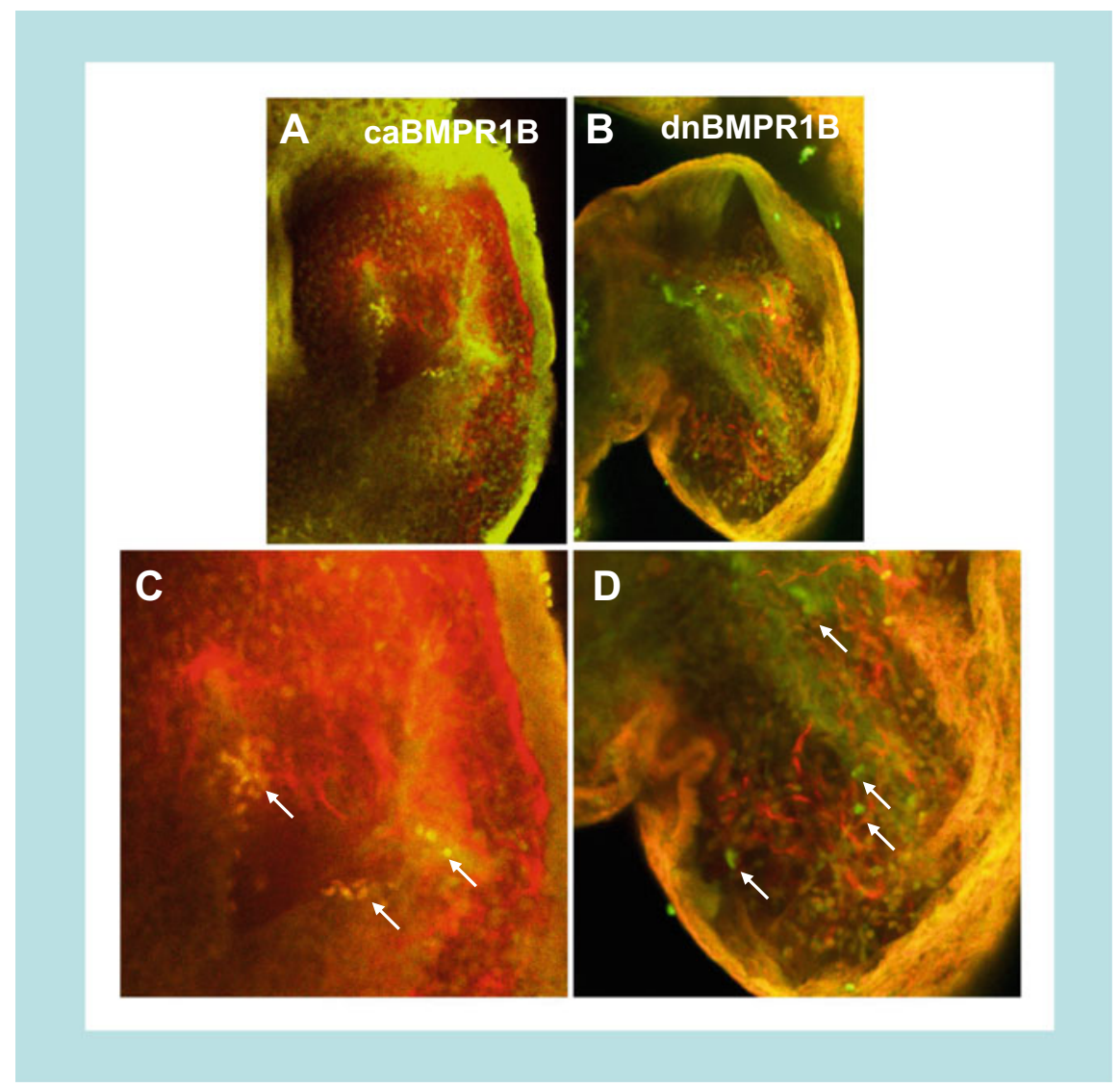

Fig. 17.5 AV cushions were microinjected with caBMPR-1B virus (a) or dnBMPR-1B virus. Cushion mesenchyme microinjected with dnBMPR-1B virus showed a significant decrease in periostin immunostaining ( red colour in b). Conversely, infection with caBMPR-1B virus showed significant increase periostin expression in cushion mesencyme (red colour in a). High magnification view of virus-infected cushion mesenchyme (c and d). In the caBMPR-1B infected cushion, there was extensive overlapping of viral marker expression (green) and periostin expression (red) (yellow arrows in c). Although periostin staining ( $r e d)$ was detected in the dnBMPR-1B infected cushion, there was little overlapping of periostin expression (red) and viral marker expression (green arrows in $\mathbf{d}$ ) 
effectively intensified by BMP-2 or caBMPR-1B. Based on these findings, it can be said that the effects of dnBMPR-1B treatment on CMC migration and phosphorSmad1/5/8 expression are as profound as the effects of noggin treatment. Moreover, caBMPR-1B treatment enhances CMC migration as much as BMP2 treatment does (Fig. 17.5). These facts strongly suggest that BMP signaling plays an important role in mesenchymal cell migration after EMT. We also showed that expressions of Id and Twist-1, which are important transcriptional factors involved in cell migration, are enhanced by BMP signaling [4].

\subsubsection{BMP2 Induces Expression of ECM Proteins in the Post-EMT Cushion}

We showed the expression patterns of three major ECM components, periostin, versican, and hyaluronic acid (HA), during AV cushion expansion and maturation. We also elucidated the role of BMP2 in the production of periostin, versican, and HA. Using a well-defined 3D CMC aggregate by hanging-drop culture on hydrated collagen gels, we found that BMP2 induces production of periostin, versican, and $\mathrm{HA}$ and that these ECM components contribute to BMP2-supported CMC migration during post-EMT cushion expansion and maturation [7].

Taken together, BMP signaling plays a critical role for AV cushion formation and AV valve maturation.

\subsection{The Role of BMP2 for Cardiomyocytes Formation}

It is well known that the BMP signaling pathway plays a central role in cardiomyogenesis [8-10]. In the past, it was found that administration of BMP-2 counteracts apoptosis of neonatal cardiomyocytes in culture, induced by serum starvation [9]. Moreover, BMP10 expression is restricted to cardiomyocytes in the developing and postnatal heart. Ligands of BMP10 have specificity for ALK1, ALK6, and BMPR2 receptors. Global disruption of BMP10 is embryonic lethal with severely impaired cardiomyocyte proliferative capacity [11]. BMP2 and BMP10 appear to control cardiomyocyte differentiation through activation of transcription factors NKX2.5, MEF2C, and TBX20 [12].

Chakraborty et al. reported that cardiomyocyte-specific Tbx20 overexpression beginning in the fetal period is sufficient for promoting cardiomyocyte proliferation [12].

Therefore, BMP signaling plays special role in linking AV cushion and cardiomyocyte formation. 


\subsection{Future Direction}

At present, many aspects of the molecular mechanisms underlying RV-TV dysplastic syndrome remain to be elucidated. To fully describe the morphogenetic mechanisms underlying TV-RV dysplastic syndrome, we need to determine the molecular mechanisms underlying the interaction between TV and RV myocardium through BMP signaling. Moreover, we need to outline the steps involved in cardiac valve and myocardium morphogenesis.

What is the next target molecule? Here, I would like to discuss about the potential of the "Notch" signaling pathway. The Notch signaling pathway plays multiple roles in cardiac morphogenesis, including regulation of valve formation, outflow tract development, and cardiac chamber maturation [13]. Notch activation is upstream of ephrin- and neuregulin-based modulation of trabeculation and BMP-2 and BMP-10 modulation of cardiomyocyte proliferation. Consistent with the involvement of Notch signaling in multiple aspects of cardiac development, components of the Notch pathway show dynamic spatial and temporal expression patterns in the developing vertebrate heart, and both endocardial and myocardial expression have been observed. Furthermore, the cross reaction between NOTCH and BMP signaling is a well-known fact [14]. Therefore, understanding the complicity of these signaling pathways is imperative for elucidating the relationship between cardiac valve and myocardium formation.

Acknowledgments Special thanks to Dr. Yukiko Sugi and Prof. Roger R. Markwald for their generous support in the experiments and excellent mentorship.

Open Access This chapter is distributed under the terms of the Creative Commons AttributionNoncommercial 2.5 License (http://creativecommons.org/licenses/by-nc/2.5/) which permits any noncommercial use, distribution, and reproduction in any medium, provided the original author(s) and source are credited.

The images or other third party material in this chapter are included in the work's Creative Commons license, unless indicated otherwise in the credit line; if such material is not included in the work's Creative Commons license and the respective action is not permitted by statutory regulation, users will need to obtain permission from the license holder to duplicate, adapt or reproduce the material.

\section{References}

1. Inai K. Encyclopedia of molecular mechanisms of disease. Ebstein's anomaly. Berlin: Springer; 2009. p. 222-3.

2. Uhl HSM. A previously undescribed cardiac malformation of the heart: almost total absence of the myocardium of the right ventricle. Bull Johns Hopkins Hosp. 1952;91:197-209.

3. Kanjuh VI, Stevenson JE, Amplatz K, Edwards JE. Congenitally unguarded tricuspid orifice with coexistent pulmonary atresia. Circulation. 1964;30:911-7.

4. Inai K, Norris RA, Hoffman F, Markwald RR, Sugi Y. BMP-2 induces cell migration and periostin expression during atrioventricular valvulogenesis. Dev Biol. 2008;315:383-96. 
5. Sugi Y, Yamamura H, Okagawa H, Markwald RR. Bone morphogenetic protein-2 can mediate myocardial regulation of atrioventricular cushion mesenchymal cell formation in mice. Dev Biol. 2004;269:505-18.

6. Okagawa H, Markwald RR, Sugi Y. Functional BMP receptor in endocardial cells is required in atrioventricular cushion mesenchymal cell formation in chick. Dev Biol. 2007;306 (1):179-92.

7. Inai K, Burnside JL, Hoffman S, Toole BP, Sugi Y. BMP-2 Induces versican and hyaluronan that contribute to Post-EMT AV cushion cell migration. PLos ONE 2012;8(10):e77593. doi: 10.1371 .

8. Zhang H, Bradley A. Mice deficient for BMP-2 are nonviable and have defects in amnion/ chorion and cardiac development. Development. 1996;122:2977-86.

9. Izumi M, Fujio Y, Kunisada K, Negoro S, Tone E, Funamoto M, et al. Bone morphogenetic protein-2 inhibits serum deprivation-induced apoptosis of neonatal cardiac myocytes through activation of the Smad1 pathway. J Biol Chem. 2001;276:31133-41.

10. Ghosh-Choudhury N, Abboud SL, Chandrasekar B, Ghosh CG. BMP-2 regulates cardiomyocyte contractility in a phosphatidylinositol 3 kinase-dependent manner. FEBS Lett. 2003;544:181-4.

11. Huang J, Elicker J, Bowens N, Liu X, Chen L, Cappola TP, et al. Myocardin regulates BMP10 expression and is required for heart development. J Clin Invest. 2012;122:3678-91.

12. Chakraborty S, Sengupta A, Yutzey KE. Tbx20 promotes cardiomyocyte proliferation and persistence of fetal characteristics in adult mouse hearts. J Mol Cell Cardiol. 2013;62:203-13.

13. Samsa LA, Yang B, Liu J. Embryonic cardiac chamber maturation: trabeculation, conduction, and cardiomyocyte proliferation. Am J Med Genet Part C Semin Med Genet. 2013;163C:157-68.

14. MacGrogan D, Luxán G, de la Pompa JL. Genetic and functional genomics approaches targeting the Notch pathway in cardiac development and congenital heart disease. Brief Funct Genom. 2014;13(1):15-27. 